\title{
High Rates of Malaria Microscopy but Low Turnaround of Test-results Among Inpatients in Tertiary Care: With Delayed Initiation, Monotherapy and Incomplete Dosing of Antimalarials
}

Ronald Kiguba ( $\nabla$ kiguba@gmail.com )

Makerere University College of Health Sciences https://orcid.org/0000-0002-2636-4115

Charles Karamagi

Clinical Epidemiology Unit, Makerere University College of Health Sciences

Sheila M. Bird

MRC Biostatistics Unit

\section{Research}

Keywords: Antimalarials, antimalarial use, delayed initiation of antimalarials, missed doses, malaria microscopy, malaria diagnosis, missed day 1 dosing, risk factors, monotherapy, incomplete dosing

Posted Date: May 27th, 2020

DOI: https://doi.org/10.21203/rs.3.rs-30045/v1

License: () (1) This work is licensed under a Creative Commons Attribution 4.0 International License. Read Full License

Version of Record: A version of this preprint was published at Malaria Journal on April 9th, 2021. See the published version at https://doi.org/10.1186/s12936021-03712-3. 


\section{Abstract}

Objectives:To describe the patterns of malaria diagnosis and antimalarial use: monotherapy, missed Day 1 dosing and risk-factors.

Methods: Prospective cohort of consented adult inpatients on the medical and gynaecological wards of Uganda's 1790-bed Mulago National Referral Hospital.

Results: One in five (19\%, 146/762; $95 \%$ confidence interval (CI): $16 \%$ to $22 \%)$ inpatients had an admitting or discharge malaria diagnosis or both. Microscopy was requested in $77 \%$ (108/141; $95 \% \mathrm{Cl}$ : $69 \%$ to $83 \%)$ of inpatients with an admitting malaria diagnosis; results were available for $46 \%$ (50/108; $95 \%$ Cl: $37 \%$ to $56 \%)$, of whom $42 \%$ (21/50; $95 \% \mathrm{Cl}$ : $28 \%$ to $57 \%$ ) were positive. Artesunate (AS) only $(47 \%, 47 / 100 ; 95 \%$ Cl: $37 \%$ to $57 \%)$ was the most frequently hospitaladministered antimalarial followed by quinine (Q) only (23\%, $95 \% \mathrm{Cl}: 15 \%$ to $32 \%)$. A quarter $(25 \%, 25 / 100 ; 95 \%$ Cl: $17 \%$ to $35 \%)$ of the inpatients missed their Day 1 dose of hospital-initiated antimalarials. Nearly half (47\%, $95 \%$ Cl: $34 \%$ to $61 \%)$ of 57 inpatients on AS and $18 \%(95 \%$ Cl: $4 \%$ to $32 \%)$ of 28 inpatients on Q missed at least one day of dosing in Days 1-3. Number of admitting diagnoses was a significant risk-factor for missed Day 1 dosing of hospital-initiated antimalarials $(\mathrm{OR}=2.7,95 \% \mathrm{Cl}: 1.53-4.54 ;$-value $<0.001)$.

Conclusions: Half the malaria microscopy results were not available; yet, the rate of testing was high. Improvement in laboratory services, procurement, prescription, dispensing and administration of antimalarials could curb missed treatment for confirmed malaria cases, monotherapy and delayed doses.

\section{Background}

Around 405,000 people died from malaria globally in 2018, 94\% of whom were from the World Health Organization (WHO) African Region. Prompt detection and appropriate treatment of malaria prevents severe disease and death [1]. The risk of mortality from severe malaria is highest during the first 24 hours of hospitalization [2]. Yet, in most moderate- to high-malaria-transmission settings, long transit-time to a suitable health facility where appropriate intravenous antimalarials can be administered could delay the initiation of appropriate antimalarials and increase the risk of patient deterioration or death ${ }^{2}$. Other impediments to the timely initiation of appropriate antimalarials include the lack of timely laboratory diagnosis and drug stock-outs [2,3].

World Health Organization recommends confirmation of malaria diagnosis by quality microscopy or malaria rapid diagnostic testing within 2 hours of patient presentation and before administration of antimalarials. Otherwise, the decision to treat should be taken on clinical grounds. If severe malaria is suspected, parasitological diagnosis should not delay initiation of antimalarials [2]. Adults with severe malaria, including pregnant women in all trimesters and breastfeeding mothers, should be treated with three doses of injectable artesunate (AS) for 24 hours minimum at 0,12 and 24 hours regardless of whether the patient can tolerate oral treatment earlier. If unable to take oral medication, the patient should continue with injectable AS once daily, for a maximum of 7days. If injectable AS is not available, once daily injectable artemether (AT) or 8-hourly quinine (Q) should be administered. Following injectable antimalarials, a full 3-day course (six doses) of oral artemisinin-based combination therapy (ACT) - mainly artemether-lumefantrine (AL) for Uganda - should be administered if the patient is able to take oral medication [2, 4-6]. Other recommended ACTs include artesunate-amodiaquine (AQ) and dihydroartemisininpiperaquine (DP). If full treatment for severe malaria is not possible at a given health facility but injectables are available, adults and children should be given one intramuscular dose of AS or Q and referred to a suitable facility for appropriate management [2].

It is necessary for patients with severe malaria to access timely appropriate antimalarials, avoid antimalarial monotherapy and complete full courses of prescribed antimalarials, which promotes therapeutic success, reduces malaria-related mortality and prevents drug resistance [7-9]. However, SSA patients with severe malaria frequently receive incomplete doses of prescribed antimalarials, or monotherapy, and/or treatment meant for uncomplicated malaria [8, 10]. We have previously shown that one-third of hospitalized patients in our setting missed Day 1 of prescribed antibiotics [3], but similar data are scarce on antimalarial use. We aim to describe the patterns of malaria diagnosis and antimalarial use by extent of use, missed opportunity for treatment, frequency of administered-treatment, medication-use-cycle (prescription-dispensing-administration), missed-dose days (including missed Day 1 dosing) and mortality. We also evaluate patient-level risk-factors for missed Day 1 dosing of prescribed antimalarials and the relationship between missed Day 1 dosing of prescribed antimalarials and length of hospital stay among adult inpatients at Mulago Hospital.

\section{Methods}

Detailed account of the study design, setting, data collection and data management is documented elsewhere [3]. Briefly, key details are the following.

\section{Study design and setting}

A prospective cohort study was conducted among inpatients, 18 years and older, at Mulago National Referral Hospital with bed capacity of 1,790 and an annual inpatient turnover of 140,000 patients. Three medical wards and one Gynaecological ward were included, each with an official bed capacity of 54 and average of 5-25 patient admissions per day [3].

Details on the prescription, dispensing and administration of medicines are hand-recorded in the patients' charts. Hospital pharmacists dispense the prescribed free-of-charge injectable and/or oral antimalarials, as appropriate, to inpatients/caregivers in quantities that discourage misuse of medicines. However, the inpatients/caregivers are instructed to return to the pharmacy early enough for more medication to avoid missing treatment ${ }^{3}$.

\section{Data collection}

The data were collected in December 2013 to April 2014 by trained research assistants. Study patients provided written informed consent and were enrolled using a systematic sampling procedure following a daily random start from the first two (Infectious Diseases and Gastrointestinal IIInesses ward), three (Haematology, Neurology and Endocrinology ward) and four (Cardiovascular, Pulmonology and Nephrology ward \& Gynaecology ward) new admissions; and 
subsequently every second, third and fourth admission, respectively. Patients were assessed at baseline (demographics, clinical conditions, medications) and on a daily basis (clinical conditions, medications) until discharge, transfer, death, or loss to follow-up. The data were collected daily from 8.00 am to 6.00 pm from Monday to Friday and from10.00am to 6.00 pm on weekends and public holidays [3].

\section{Data management}

Epidata 3.1 software was programmed with checks to limit data entry errors and the electronic database password-secured to limit access only to authorized personnel.

\section{Statistical analysis}

\section{Patterns of malaria diagnosis and antimalarial use}

The proportions of inpatients who received antimalarials preadmission and during hospitalization were determined using, as numerator, the number of inpatients who received at least one antimalarial and, as denominator, the total number of study patients. We calculated proportions of inpatients who had malaria microscopy done and those who experienced antimalarial missed-dose days, including missed Day 1 dosing. See Appendix for details on time-to-firstdose and parenteral-to-oral-switch of antimalarials.

We used Chi-squared tests to screen univariate-level relationships between patient-level characteristics and antimalarial use during hospitalization (yes/no); and potential patient-level risk-factors for missed Day 1 antimalarials during hospitalization. Logistic regression was used to identify risk-factors for missed Day 1 antimalarials. Results were expressed as odds ratios (ORs) with their $95 \%$ confidence intervals (Cls). Poisson Cls were used for counts below 16 . Stata 14.0 [11] was used for all the analyses.

\section{Identification of missed Day 1 dosing of antimalarials}

Among inpatients for whom an antimalarial was prescribed during hospitalization and at least one dose administered, missed Day 1 dosing was measured in two ways; i) calendar-day as proposed by Kiguba et al 2016 [3], see Appendix, and ii) 24-hour timescale using date-and-time of hospital admission and dateand-time of first in-hospital antimalarial dose.

\section{Results}

\section{Study population}

Demographic and clinical characteristics:The median age of 762 inpatients was 30 years (interquartile range, IQR, 24 to 42 years), see Table 1.0 ne in five (19\%, 141/762; $95 \%$ Cl: $16 \%$ to $21 \%)$ inpatients had an admittingmalariadiagnosis,see Tables 1 \& 2: $11 \%(16 / 141 ; 95 \%$ Cl: $7 \%$ to $18 \%)$ had malaria as their single admitting diagnosis. One in eight (12\%, 88/762; $95 \% \mathrm{Cl}$ : $9 \%$ to $14 \%)$ inpatients had a discharge malaria diagnosis: $44 \%$ (39/88; $95 \% \mathrm{Cl}: 34 \%$ to $55 \%)$ had malaria as their single discharge diagnosis. One in five (19\%, 146/762; $95 \% \mathrm{Cl}$ : $16 \%$ to $22 \%)$ had an admitting or discharge malaria diagnosis or both, see Table 2:21\% (30/146; $95 \%$ Cl: $14 \%$ to $28 \%)$ had malaria-in-pregnancy. 
Table 1

Demographic and clinical characteristics of 762 inpatients, Uganda

\section{Characteristic}

Age, years

Length of hospital stay, days ${ }^{a}$

Patient-days of observation

Extent of antimalarial use

\begin{tabular}{llll} 
& \multicolumn{3}{l}{ Antimalarial Use during the Current Hospitalizatic } \\
& $\mathbf{n}(\%)$ & Total \\
\hline Pre-admissionantimalarials & Yes & No & 762 \\
\hline In-hospitalantimalarials & $97(13)$ & $665(87)$ & 762 \\
\hline Pre-admission antimalarials & $100(13)$ & $662(87)$ & 100 \\
\hline Pre-/in-hospital co-trimoxazole & $38(38)$ & $62(62)$ & 100 \\
\hline In-hospital antibiotics & $15(15)$ & $87(87)$ & 100 \\
\hline In-hospital antiretrovirals & $61(61)$ & $39(39)$ & 100 \\
\hline Pre-/in-hospital antimalarials & $14(14)$ & $86(86)$ & 762
\end{tabular}

Subgroup analyses on key variables

\section{Antimalarial Use, n (\%)}

Yes No
Overall

$30(24-42)$

$4(3-6)$

3,741

$\begin{array}{lll}\text { Yes } & \text { No } & \text { Overall } \\ 27(21-35) & 30(25-43) & 30(24-42) \\ 4(3-5) & 4(3-6) & 4(3-6) \\ 454 & 3,287 & 3,741\end{array}$




\begin{tabular}{|c|c|c|c|c|c|c|}
\hline Positive & $23(10)$ & $209(90)$ & $232[30]$ & 0.7 & $0.39-1.11$ & 0.113 \\
\hline Unknown & $28(15)$ & $162(85)$ & $190[25]$ & 1.0 & $0.62-1.70$ & 0.919 \\
\hline \multicolumn{7}{|c|}{ Hospitalization in past 3-months } \\
\hline No & $75(14)$ & $455(86)$ & $532[70]$ & 1.0 & & \\
\hline Yes & $25(11)$ & $205(89)$ & $230[30]$ & 0.7 & $0.46-1.20$ & 0.227 \\
\hline \multicolumn{7}{|c|}{ Charlson's co-morbidity index score } \\
\hline Zero & $64(16)$ & $329(84)$ & $393[52]$ & 1.0 & & \\
\hline One or more & $36(10)$ & $333(90)$ & $369[48]$ & 0.6 & $0.36-0.86$ & 0.008 \\
\hline \multicolumn{7}{|c|}{ Antiretroviral therapy use } \\
\hline No & $86(14)$ & $549(86)$ & $635[83]$ & 1.0 & & \\
\hline Yes & $14(11)$ & $113(89)$ & $127[17]$ & 0.8 & $0.43-1.44$ & 0.444 \\
\hline \multicolumn{7}{|c|}{ Microscopy - Malaria Parasitaemia Results Available } \\
\hline No & $62(62)$ & $616(93)$ & 678 [89] & 1.0 & & \\
\hline Yes & $38(38)$ & $46(7)$ & $84[11]$ & 8.2 & $4.81-14.0$ & $<0.001$ \\
\hline \multicolumn{7}{|c|}{ Major admitting diagnosis } \\
\hline \multicolumn{7}{|l|}{ Malaria } \\
\hline No & $17(3)$ & $604(97)$ & $621[81]$ & 1.0 & & \\
\hline Yes & $83(59)$ & $58(41)$ & $141[19]$ & 50 & 28.3-91.5 & $<0.001$ \\
\hline \multicolumn{7}{|c|}{ Immunosuppressed syndrome (ISS) or HIV/AIDSe } \\
\hline No & $86(14)$ & $524(86)$ & $610[80]$ & 1.0 & & \\
\hline Yes & $14(9)$ & $14(91)$ & $152[20]$ & 0.6 & $0.34-1.12$ & 0.113 \\
\hline \multicolumn{7}{|c|}{ Tuberculosis (TB) } \\
\hline No & $92(14)$ & $548(86)$ & $640[84]$ & 1.0 & & \\
\hline Yes & $8(7)$ & $114(93)$ & $122[16]$ & 0.4 & $0.20-0.88$ & 0.023 \\
\hline \multicolumn{7}{|c|}{ Sepsis-related working diagnosis } \\
\hline No & $81(12)$ & $597(88)$ & 678 [89] & 1.0 & & \\
\hline Yes & $19(23)$ & $65(77)$ & $84[11]$ & 2.2 & $1.23-3.78$ & 0.007 \\
\hline \multicolumn{7}{|c|}{ Respiratory Conditions except TB } \\
\hline No & $85(13)$ & $547(87)$ & $632[83]$ & 1.0 & & \\
\hline Yes & $15(12)$ & $115(88)$ & $130[17]$ & 0.8 & $0.47-1.51$ & 0.557 \\
\hline \multicolumn{7}{|c|}{ Miscellaneous infections } \\
\hline No & $78(12)$ & $571(88)$ & $649[85]$ & 1.0 & & \\
\hline Yes & $22(19)$ & $91(81)$ & $113[15]$ & 1.8 & $1.05-2.98$ & 0.032 \\
\hline
\end{tabular}


Table 2

Malaria detection by laboratory diagnosis among 762 hospitalized

Malaria suspected at admission

Malaria not susp

Malaria at discharge $(n=83)$

No malaria at discharge $(n=58)$

Malaria at disch

Microscopy requested, $\mathrm{n}(\%)^{¥}$

$\begin{array}{llllllll}\text { Yes } & & \text { No } & \text { Yes } & & \text { No } \\ \begin{array}{l}\text { Returned } \\ \text { Positive }\end{array} & \begin{array}{l}\text { Returned } \\ \text { Negative }\end{array} & \begin{array}{l}\text { Not } \\ \text { Returned }\end{array} & \text { Not Requested } & \begin{array}{l}\text { Returned } \\ \text { Positive }\end{array} & \begin{array}{l}\text { Returned } \\ \text { Negative }\end{array} & \text { Not Returned } & \text { Not Requested } \\ 21(25) & 13(16) & 31(37) & 18(22) & 0(0) & 16(28) & 27(47) & 15(26) \\ \text { Rositive }\end{array}$

In-hospital administration of antimalarials, $\mathbf{n}(\%)$

$\begin{array}{lllllllllllllllllll}\text { Yes } & \text { No } & \text { Yes } & \text { No } & \text { Yes } & \text { No } & \text { Yes } & \text { No } & \text { Yes } & \text { No } & \text { Yes } & \text { No } & \text { Yes } & \text { No } & \text { Yes } & \text { No } & \text { Yes } & \text { No } \\ 20 & 1(5) & 11(85) & 2(15) & \begin{array}{l}26 \\ (84)\end{array} & 5(16) & 15(83) & 3(17) & 0(0) & 0(0) & 5(31) & 11(69) & 3 & 24(89) & 3(20) & 12(80) & 1 & 0(0) \\ (95) & & & & & & & & & & & (11) & & \end{array}$

Single admitting diagnosis of malaria, $\mathrm{n}(\%)$

\begin{tabular}{|c|c|c|c|c|c|c|c|c|c|c|c|c|c|c|c|c|c|}
\hline Yes & No & Yes & No & Yes & No & Yes & No & Yes & No & Yes & No & Yes & No & Yes & No & Yes & No \\
\hline $\begin{array}{l}5 \\
(24)\end{array}$ & $\begin{array}{l}16 \\
(76)\end{array}$ & م & $\begin{array}{l}13 \\
(100)\end{array}$ & $\begin{array}{l}5 \\
(16)\end{array}$ & $\begin{array}{l}26 \\
(84)\end{array}$ & 2) & $\begin{array}{l}14 \\
(78)\end{array}$ & $\begin{array}{l}0 \\
(0)\end{array}$ & $\begin{array}{l}0 \\
(0)\end{array}$ & $1(6)$ & $\begin{array}{l}15 \\
(94)\end{array}$ & $\begin{array}{l}1 \\
\text { (4) }\end{array}$ & $\begin{array}{l}26 \\
(96)\end{array}$ & $0(0)$ & $\begin{array}{l}15 \\
(100)\end{array}$ & (0) & $\begin{array}{l}1 \\
(100)\end{array}$ \\
\hline
\end{tabular}

Received antimalarials during the 4-weeks preadmission, $\mathrm{n}(\%)$

$\begin{array}{llllllllllllllllll}\text { Yes } & \text { No } & \text { Yes } & \text { No } & \text { Yes } & \text { No } & \text { Yes } & \text { No } & \text { Yes } & \text { No } & \text { Yes } & \text { No } & \text { Yes } & \text { No } & \text { Yes } & \text { No } & \text { Yes } & \text { No } \\ 7 & 14 & 5(38) & 8 & 8 & 23 & 7(39) & 11 & 0 & 0 & 5 & 11 & 8 & 19 & 3 & 12 & 0(0) & 1 \\ (33) & (67) & & (62) & (26) & (74) & & (61) & (0) & (0) & (31) & (69) & (30) & (70) & (20) & (80) & & (100)\end{array}$

Single discharge diagnosis of malaria, $n(\%)$

\begin{tabular}{|c|c|c|c|c|c|c|c|c|c|c|c|c|c|c|c|c|c|}
\hline Yes & No & Yes & No & Yes & No & Yes & No & Yes & No & Yes & No & Yes & No & Yes & No & Yes & No \\
\hline $\begin{array}{l}9 \\
\text { (43) }\end{array}$ & $\begin{array}{l}12 \\
(57)\end{array}$ & $3(23)$ & $\begin{array}{l}10 \\
(77)\end{array}$ & $\begin{array}{l}16 \\
(52)\end{array}$ & $\begin{array}{l}15 \\
(48)\end{array}$ & $\begin{array}{l}11 \\
(61)\end{array}$ & $\begin{array}{l}7 \\
(39)\end{array}$ & $\begin{array}{l}0 \\
(0)\end{array}$ & $\begin{array}{l}0 \\
(0)\end{array}$ & $0(0)$ & $\begin{array}{l}16 \\
(100)\end{array}$ & $\begin{array}{l}0 \\
(0)\end{array}$ & $\begin{array}{l}27 \\
(100)\end{array}$ & $\begin{array}{l}0 \\
(100)\end{array}$ & $15(0)$ & $0(0)$ & $\begin{array}{l}1 \\
(100)\end{array}$ \\
\hline
\end{tabular}

${ }^{ \pm} 19 \%$ (146/762; $95 \%$ confidence interval (Cl): 16\% to 22\%) of inpatients had either an admitting or discharge malaria diagnosis or both; $19 \%$ (141/762; $95 \%$ C requests had concurrent malaria rapid diagnostic testing (mRDT) done. However, mRDT results were available for only one inpatient, who tested positive.

Laboratory diagnosis of malaria:Microscopy was requested in 26\% (201/762) of inpatients; laboratory results were available for $42 \%$ (84/201; $95 \%$ Cl: $34 \%$ to $48 \%$ ) of them, $30 \%$ (25/84; $95 \% \mathrm{Cl}$ : $20 \%$ to $41 \%)$ tested positive. Microscopy was requested in $77 \%(108 / 141 ; 95 \%$ Cl: $69 \%$ to $83 \%)$ of inpatients with an admitting malaria diagnosis; laboratoryresults were available for $46 \%(50 / 108$; $95 \% \mathrm{Cl}$ : $37 \%$ to $56 \%)$ of them, $42 \%(21 / 50 ; 95 \%$ Cl: $28 \%$ to $57 \%)$ tested positive, see Appendix.At bivariate level, inpatients with an admitting malaria-in-pregnancy diagnosis were ten-fold more likely to test positive for malaria when compared with non-pregnancy-related malaria inpatients (odds ratio, $\mathrm{OR}=10.1 ; 95 \% \mathrm{Cl}: 1.55$ to $65.96 ; 1$ degree of freedom, df; $\chi 2=9 ; P$-value $=0.003$ ) i.e. [ $82 \%(9 / 11 ; 95 \%$ Cl: $48 \%$ to $98 \%)$ vs. $31 \%(12 / 39 ; 95 \%$ Cl: $17 \%$ to $48 \%)$, respectively.

\section{Extent of antimalarial use}

Thirteen percent (97/762; $95 \% \mathrm{Cl}: 10 \%$ to $15 \%$ ) of inpatients received antimalarials during the 4-weekspre-admission, see Table 1:of whom 44\% (43/97; $95 \%$ Cl: $34 \%$ to $55 \%$ ) had an admitting malariadiagnosis. Thirteen percent (100/762; $95 \% \mathrm{Cl}: 11 \%$ to $16 \%$ ) of inpatients received antimalarials during the current hospitalization, see Table 1:of whom 83\% (83/100; $95 \% \mathrm{Cl}$ : 74\% to 90\%) had an admitting malaria diagnosis, see Table 1.

\section{Missed opportunity for hospital-initiated antimalarials}

Four of 25 (16\%, 95\% Cl: $5 \%$ to $36 \%)$ inpatients with a positive malaria test did not receive in-hospital antimalarials; none of the fourdied while in hospital, see Box 1, and none had malaria-in-pregnancy.

\section{Frequency of administered antimalarials}

Four-week preadmission period:At patient-level,oral artemether-lumefantrine (AL) only (52\%, 50/97; $95 \%$ Cl: $41 \%$ to $62 \%$ ) was most frequently administered followed by injectablequinine (Q) only (23\%, 22/97; $95 \%$ Cl: $15 \%$ to $32 \%)$, see Table 3 and Appendix.

Current hospitalization:At patient-level, AS only (47\%, 47/100; $95 \%$ Cl: $37 \%$ to $57 \%$ ) was the most frequently administered followed by Q only (23\%, $23 / 100$; $95 \% \mathrm{Cl}: 15 \%$ to $32 \%)$, AL only (15\%, 15/100; $95 \% \mathrm{Cl}$ : $9 \%$ to $24 \%)$ and AS + AL only (8\%, 8/100; $95 \%$ Cl: $4 \%$ to $15 \%)$, among others; see Table 3 and Appendix. 


\begin{tabular}{|c|c|c|}
\hline Antimalarial & Number: $\mathrm{n}, \%$ & \\
\hline \multicolumn{3}{|l|}{ Patient-level } \\
\hline \multicolumn{3}{|l|}{ Pre-admission, $n=97$} \\
\hline Artemether-Lumefantrine only & 50 & $52 \%$ \\
\hline Quinine only & 22 & $23 \%$ \\
\hline Sulfadoxine-Pyrimethamine only & 9 & $9 \%$ \\
\hline Artesunate only & 5 & $5 \%$ \\
\hline Coartem + Quinine only & 4 & $4 \%$ \\
\hline Duocotexcin only & 2 & $2 \%$ \\
\hline Artemether only & 1 & $1 \%$ \\
\hline Artesunate + Duocotexcin only & 1 & $1 \%$ \\
\hline P-alaxin + Quinine only & 1 & $1 \%$ \\
\hline Artemether + Quinine + Doxycycline only & 1 & $1 \%$ \\
\hline Dihydroartemisinin-Piperaquine only & 1 & $1 \%$ \\
\hline \multicolumn{3}{|l|}{ In-hospital, $n=100$} \\
\hline Artesunate only ${ }^{ \pm}$ & 47 & $47 \%$ \\
\hline Quinine only & 23 & $23 \%$ \\
\hline Artemether-Lumefantrine only & 15 & $15 \%$ \\
\hline Artesunate + Artemether-Lumefantrine only & 8 & $8 \%$ \\
\hline Quinine + Artemether/Lumefantrine only & 3 & $3 \%$ \\
\hline Sulfadoxine-Pyrimethamine only & 2 & $2 \%$ \\
\hline Artesunate + Quinine only & 2 & $2 \%$ \\
\hline \multicolumn{3}{|l|}{ Drug-level } \\
\hline \multicolumn{3}{|l|}{ Pre-admission, $n=105$} \\
\hline Artemether-Lumefantrine & 54 & $51 \%$ \\
\hline Quinine & 28 & $27 \%$ \\
\hline Sulfadoxine-Pyrimethamine & 9 & $9 \%$ \\
\hline Artesunate & 6 & $6 \%$ \\
\hline Duocotexcin & 3 & $3 \%$ \\
\hline Artemether & 2 & $2 \%$ \\
\hline Dihydroartemisinin-Piperaquine & 2 & $2 \%$ \\
\hline Doxycycline & 1 & $1 \%$ \\
\hline \multicolumn{3}{|l|}{ In-hospital, $n=113$} \\
\hline Artesunate & 57 & $50 \%$ \\
\hline Quinine & 28 & $25 \%$ \\
\hline Artemether-Lumefantrine & 26 & $23 \%$ \\
\hline Sulfadoxine-Pyrimethamine & 2 & $2 \%$ \\
\hline
\end{tabular}

Medication-use-cycle 


\section{Overview of prescription, dispensing and administration of antimalarials}

Overall: Antimalarials were prescribed for 15\% (114/762) of inpatients, dispensed to 79\% (90/114), yet, administered in 100 inpatients (93 of 100 had an antimalarial prescription), see Appendix for details on AS, Q and AL.

\section{Incomplete dosing of in-hospital antimalarials}

Artesunate.25\% (14/57; $95 \% \mathrm{Cl}$ : $14 \%$ to $38 \%)$ of inpatients in whom in-hospital AS was administeredreceived<3 doses of both dispensed and administered AS irrespective of pregnancy, see Appendix.

Quinine: $21 \%(6 / 28 ; 95 \% \mathrm{Cl}$ : $8 \%$ to $41 \%)$ of inpatients in whom in-hospital Q was administeredreceived $<3$ doses of both dispensed and administered Q irrespective of pregnancy, see Appendix.

Artemether-Lumefantrine: $71 \%$ (20/28; $95 \%$ Cl: $51 \%$ to $87 \%)$ of inpatients in whom in-hospital AL was administeredreceived $<6$ doses of administered AL.

\section{Missed Day 1 dosing of hospital-prescribed antimalarials}

Calendar-day: A quarter (25\%, 25/100; $95 \%$ Cl: $17 \%$ to 35\%) of inpatients who received antimalarials during the current hospitalization missed their Day 1 dose of hospital-initiated antimalarials based on calendar-day. Similar results of missed Day 1 dosing were obtainedbased on post-admission 24-hour-delay, see Appendix.

\section{Missed-dose days of the most frequently administered hospital-prescribed antimalarials}

Artesunate:Around a quarter (28\%, 16/57; $95 \%$ Cl: $17 \%$ to $42 \%)$ of inpatients who initiated antimalarials with AS missed their Day 1 dose(s) based on calendarday; $19 \%$ (10/52; $95 \%$ Cl: $10 \%$ to $32 \%$ ) missed Day 2 and $27 \%$ (8/30; $95 \%$ Cl: $12 \%$ to $46 \%)$ missed Day 3 . Nearly half (47\%, $27 / 57 ; 95 \%$ Cl: $34 \%$ to $61 \%)$ the inpatients missed at least one day of AS dosing in Days 1-3, see Table 4 
Table 4

Missed dose-days of artesunate injection among 57 hospitalized patients who received in-hospital intravenous artesunate, Ugand

\begin{tabular}{|c|c|c|c|c|c|c|c|c|c|c|c|c|}
\hline fileno & $\begin{array}{l}\text { Length } \\
\text { of } \\
\text { stay, } \\
\text { days }\end{array}$ & $\begin{array}{l}\text { Preadmission } \\
\text { antimalarial } \\
\text { use }\end{array}$ & $\begin{array}{l}\text { Admitting } \\
\text { MalariaDiagnosis }\end{array}$ & $\begin{array}{l}\text { Discharge } \\
\text { Malaria } \\
\text { Diagnosis }\end{array}$ & $\begin{array}{l}\text { AL co- } \\
\text { prescribed }\end{array}$ & $\begin{array}{l}\text { Switched } \\
\text { to AL }\end{array}$ & $\begin{array}{l}\text { AS Doses } \\
\text { Prescribed }\end{array}$ & $\begin{array}{l}\text { AS } \\
\text { Doses } \\
\text { Received }\end{array}$ & Day 1 & Day 2 & Day 3 & Day \\
\hline 6 & 3 & No & Yes & No & No & No & 3 & 1 & & $\begin{array}{l}1 \\
\text { dose }\end{array}$ & & \\
\hline 20 & 3 & No & Yes & Yes & Yes & No & 2 & 3 & $\begin{array}{l}1 \\
\text { dose }\end{array}$ & $\begin{array}{l}2 \\
\text { doses }\end{array}$ & & \\
\hline 75 & 4 & No & Yes & Yes & No & No & 6 & 2 & $\begin{array}{l}1 \\
\text { dose }\end{array}$ & $\begin{array}{l}1 \\
\text { dose }\end{array}$ & & \\
\hline 77 & 5 & No & Yes & Yes & Yes & No & 3 & 3 & $\begin{array}{l}1 \\
\text { dose }\end{array}$ & $\begin{array}{l}1 \\
\text { dose }\end{array}$ & $\begin{array}{l}1 \\
\text { dose }\end{array}$ & \\
\hline 90 & 2 & No & Yes & Yes & Yes & No & 2 & 1 & $\begin{array}{l}1 \\
\text { dose }\end{array}$ & & & \\
\hline 109 & 4 & Yes & Yes & Yes & Yes & No & 10 & 3 & $\begin{array}{l}1 \\
\text { dose }\end{array}$ & & $\begin{array}{l}1 \\
\text { dose }\end{array}$ & $\begin{array}{l}1 \\
\text { dose }\end{array}$ \\
\hline 112 & 5 & No & No & No & No & No & 3 & 2 & & $\begin{array}{l}2 \\
\text { doses }\end{array}$ & & \\
\hline 125 & 2 & No & Yes & Yes & Yes & Yes & 3 & 2 & $\begin{array}{l}2 \\
\text { doses }\end{array}$ & & & \\
\hline 128 & 4 & No & Yes & No & Yes & Yes & 3 & 2 & $\begin{array}{l}1 \\
\text { dose }\end{array}$ & $\begin{array}{l}1 \\
\text { dose }\end{array}$ & & \\
\hline 130 & 5 & Yes & Yes & Yes & Yes & Yes & 3 & 1 & $\begin{array}{l}1 \\
\text { dose }\end{array}$ & & & \\
\hline 169 & 4 & No & Yes & Yes & Yes & No & 3 & 2 & $\begin{array}{l}1 \\
\text { dose }\end{array}$ & $\begin{array}{l}1 \\
\text { dose }\end{array}$ & & \\
\hline 174 & 5 & No & Yes & Yes & No & No & 3 & 4 & & $\begin{array}{l}1 \\
\text { dose }\end{array}$ & $\begin{array}{l}2 \\
\text { doses }\end{array}$ & $\begin{array}{l}1 \\
\text { dose }\end{array}$ \\
\hline 215 & 5 & No & Yes & Yes & No & No & 3 & 3 & $\begin{array}{l}1 \\
\text { dose }\end{array}$ & $\begin{array}{l}1 \\
\text { dose }\end{array}$ & $\begin{array}{l}1 \\
\text { dose }\end{array}$ & \\
\hline 218 & 5 & No & Yes & No & No & No & 3 & 3 & & $\begin{array}{l}1 \\
\text { dose }\end{array}$ & $\begin{array}{l}1 \\
\text { dose }\end{array}$ & $\begin{array}{l}1 \\
\text { dose }\end{array}$ \\
\hline 233 & 10 & Yes & Yes & No & No & No & 3 & 2 & $\begin{array}{l}1 \\
\text { dose }\end{array}$ & $\begin{array}{l}1 \\
\text { dose }\end{array}$ & & \\
\hline 251 & 3 & Yes & No & No & No & No & 2 & 3 & $\begin{array}{l}1 \\
\text { dose }\end{array}$ & $\begin{array}{l}2 \\
\text { doses }\end{array}$ & & \\
\hline 253 & 5 & Yes & Yes & Yes & Yes & No & 2 & 2 & $\begin{array}{l}1 \\
\text { dose }\end{array}$ & $\begin{array}{l}1 \\
\text { dose }\end{array}$ & & \\
\hline 291 & 2 & No & Yes & Yes & Yes & No & 3 & 2 & $\begin{array}{l}1 \\
\text { dose }\end{array}$ & $\begin{array}{l}1 \\
\text { dose }\end{array}$ & & \\
\hline 294 & 3 & No & Yes & Yes & Yes & No & 2 & 3 & $\begin{array}{l}2 \\
\text { doses }\end{array}$ & $\begin{array}{l}1 \\
\text { dose }\end{array}$ & & \\
\hline 295 & 10 & No & No & No & No & No & 3 & 4 & & $\begin{array}{l}1 \\
\text { dose }\end{array}$ & $\begin{array}{l}1 \\
\text { dose }\end{array}$ & $\begin{array}{l}2 \\
\text { dose }\end{array}$ \\
\hline 299 & 3 & No & Yes & Yes & Yes & No & 3 & 2 & & $\begin{array}{l}2 \\
\text { doses }\end{array}$ & & \\
\hline 300 & 5 & No & No & Yes & Yes & No & 2 & 3 & & $\begin{array}{l}1 \\
\text { dose }\end{array}$ & $\begin{array}{l}1 \\
\text { dose }\end{array}$ & $\begin{array}{l}1 \\
\text { dose }\end{array}$ \\
\hline 303 & 2 & No & Yes & Yes & Yes & No & 4 & 1 & $\begin{array}{l}1 \\
\text { dose }\end{array}$ & & & \\
\hline 305 & 3 & Yes & Yes & Yes & Yes & No & 4 & 3 & $\begin{array}{l}1 \\
\text { dose }\end{array}$ & $\begin{array}{l}1 \\
\text { dose }\end{array}$ & $\begin{array}{l}1 \\
\text { dose }\end{array}$ & \\
\hline 313 & 3 & No & Yes & Yes & Yes & No & 3 & 3 & $\begin{array}{l}1 \\
\text { dose }\end{array}$ & $\begin{array}{l}2 \\
\text { doses }\end{array}$ & & \\
\hline
\end{tabular}

${ }^{ \pm}$The variation from 1 to 2 AS doses depends on time of day that an inpatient initiates treatment. Injectable AS is given at $0,12,24$ hourstheninpatient is swit might receive 1 to 2 AS doses per calendar-day - very rarely 3 AS doses. ${ }^{*} \mathrm{~N}=[($ The 57 artesunate users) - (Number of inpatients who did not have artesunate confidence intervals for theestimates are $28 \%(17 \%$ to $42 \%), 19 \%(10 \%$ to $32 \%), 27 \%(12 \%$ to $46 \%), 7 \%(0 \%$ to $36 \%)$ and $20 \%$ (0\% to $72 \%)$. Nearly half (47\%, 27 inpatients on AS missed at least one day of AS dosing in Days 1-3. 


\begin{tabular}{|c|c|c|c|c|c|c|c|c|c|c|c|c|}
\hline 314 & 3 & Yes & Yes & Yes & No & No & 3 & 3 & $\begin{array}{l}1 \\
\text { dose }\end{array}$ & $\begin{array}{l}1 \\
\text { dose }\end{array}$ & $\begin{array}{l}1 \\
\text { dose }\end{array}$ & \\
\hline 315 & 3 & Yes & Yes & Yes & Yes & No & 3 & 2 & $\begin{array}{l}1 \\
\text { dose }\end{array}$ & $\begin{array}{l}1 \\
\text { dose }\end{array}$ & & \\
\hline fileno & $\begin{array}{l}\text { Length } \\
\text { of } \\
\text { stay, } \\
\text { days }\end{array}$ & $\begin{array}{l}\text { Preadmission } \\
\text { antimalarial } \\
\text { use }\end{array}$ & $\begin{array}{l}\text { Admitting } \\
\text { MalariaDiagnosis }\end{array}$ & $\begin{array}{l}\text { Discharge } \\
\text { Malaria } \\
\text { Diagnosis }\end{array}$ & $\begin{array}{l}\text { AL co- } \\
\text { prescribed }\end{array}$ & $\begin{array}{l}\text { Switched } \\
\text { to AL }\end{array}$ & $\begin{array}{l}\text { AS Doses } \\
\text { Prescribed }\end{array}$ & $\begin{array}{l}\text { AS } \\
\text { Doses } \\
\text { Received }\end{array}$ & Day 1 & Day 2 & Day 3 & Day \\
\hline 320 & 5 & Yes & Yes & Yes & Yes & No & 3 & 4 & $\begin{array}{l}1 \\
\text { dose }\end{array}$ & $\begin{array}{l}1 \\
\text { dose }\end{array}$ & $\begin{array}{l}1 \\
\text { dose }\end{array}$ & $\begin{array}{l}1 \\
\text { dose }\end{array}$ \\
\hline 326 & 4 & Yes & Yes & Yes & Yes & No & 3 & 3 & $\begin{array}{l}1 \\
\text { dose }\end{array}$ & $\begin{array}{l}1 \\
\text { dose }\end{array}$ & $\begin{array}{l}1 \\
\text { dose }\end{array}$ & \\
\hline 328 & 3 & Yes & Yes & Yes & Yes & No & 3 & 3 & $\begin{array}{l}1 \\
\text { dose }\end{array}$ & $\begin{array}{l}1 \\
\text { dose }\end{array}$ & $\begin{array}{l}1 \\
\text { dose }\end{array}$ & \\
\hline 335 & 5 & Yes & Yes & Yes & Yes & No & 3 & 3 & $\begin{array}{l}1 \\
\text { dose }\end{array}$ & & $\begin{array}{l}1 \\
\text { dose }\end{array}$ & $\begin{array}{l}1 \\
\text { dose }\end{array}$ \\
\hline 348 & 7 & Yes & Yes & Yes & Yes & Yes & 3 & 3 & $\begin{array}{l}1 \\
\text { dose }\end{array}$ & & & \\
\hline 353 & 5 & No & No & No & Yes & No & 3 & 3 & & $\begin{array}{l}1 \\
\text { dose }\end{array}$ & $\begin{array}{l}1 \\
\text { dose }\end{array}$ & $\begin{array}{l}1 \\
\text { dose }\end{array}$ \\
\hline 354 & 3 & Yes & Yes & Yes & Yes & No & 3 & 2 & $\begin{array}{l}1 \\
\text { dose }\end{array}$ & $\begin{array}{l}1 \\
\text { dose }\end{array}$ & & \\
\hline 361 & 4 & No & Yes & Yes & No & No & 3 & 2 & $\begin{array}{l}1 \\
\text { dose }\end{array}$ & $\begin{array}{l}1 \\
\text { dose }\end{array}$ & & \\
\hline 385 & 2 & No & Yes & Yes & Yes & No & 3 & 1 & $\begin{array}{l}1 \\
\text { dose }\end{array}$ & & & \\
\hline 389 & 9 & No & Yes & Yes & No & No & 3 & 2 & $\begin{array}{l}1 \\
\text { dose }\end{array}$ & $\begin{array}{l}1 \\
\text { dose }\end{array}$ & & \\
\hline 400 & 6 & No & Yes & Yes & No & No & 3 & 3 & & & & $\begin{array}{l}1 \\
\text { dose }\end{array}$ \\
\hline 420 & 3 & Yes & No & No & Yes & No & 3 & 1 & $\begin{array}{l}1 \\
\text { dose }\end{array}$ & & & \\
\hline 424 & 4 & No & Yes & Yes & Yes & No & 3 & 3 & & $\begin{array}{l}1 \\
\text { dose }\end{array}$ & $\begin{array}{l}2 \\
\text { doses }\end{array}$ & \\
\hline 425 & 3 & Yes & Yes & No & Yes & No & 3 & 3 & $\begin{array}{l}1 \\
\text { dose }\end{array}$ & 2 & & \\
\hline 437 & 4 & No & Yes & Yes & No & No & 3 & 3 & $\begin{array}{l}1 \\
\text { dose }\end{array}$ & $\begin{array}{l}1 \\
\text { dose }\end{array}$ & $\begin{array}{l}1 \\
\text { dose }\end{array}$ & \\
\hline 441 & 3 & Yes & No & Yes & Yes & No & 3 & 3 & $\begin{array}{l}1 \\
\text { dose }\end{array}$ & $\begin{array}{l}2 \\
\text { doses }\end{array}$ & & \\
\hline 445 & 4 & Yes & Yes & Yes & Yes & No & 3 & 3 & & $\begin{array}{l}1 \\
\text { dose }\end{array}$ & $\begin{array}{l}2 \\
\text { doses }\end{array}$ & \\
\hline 447 & 9 & No & Yes & Yes & Yes & Yes & 4 & 3 & & & $\begin{array}{l}1 \\
\text { dose }\end{array}$ & $\begin{array}{l}1 \\
\text { dos }\end{array}$ \\
\hline 466 & 4 & Yes & Yes & No & Yes & Yes & 2 & 3 & & 2 & $\begin{array}{l}1 \\
\text { dose }\end{array}$ & \\
\hline 475 & 4 & No & Yes & Yes & Yes & No & 6 & 2 & & & $\begin{array}{l}1 \\
\text { dose }\end{array}$ & $\begin{array}{l}1 \\
\text { dose }\end{array}$ \\
\hline 520 & 3 & No & Yes & Yes & Yes & Yes & 1 & 1 & $\begin{array}{l}1 \\
\text { dose }\end{array}$ & & & \\
\hline 545 & 16 & Yes & Yes & No & No & No & 3 & 3 & $\begin{array}{l}1 \\
\text { dose }\end{array}$ & $\begin{array}{l}2 \\
\text { doses }\end{array}$ & & \\
\hline 571 & 4 & No & Yes & Yes & No & No & 3 & 1 & $\begin{array}{l}1 \\
\text { dose }\end{array}$ & & & \\
\hline 584 & 6 & No & Yes & No & No & No & 3 & 4 & $\begin{array}{l}1 \\
\text { dose }\end{array}$ & $\begin{array}{l}1 \\
\text { dose }\end{array}$ & $\begin{array}{l}2 \\
\text { doses }\end{array}$ & \\
\hline
\end{tabular}

${ }^{ \pm}$The variation from 1 to 2 AS doses depends on time of day that an inpatient initiates treatment. Injectable AS is given at $0,12,24$ hourstheninpatient is swit might receive 1 to 2 AS doses per calendar-day - very rarely 3 AS doses. ${ }^{*} \mathrm{~N}=[($ The 57 artesunate users) - (Number of inpatients who did not have artesunate confidence intervals for theestimates are $28 \%(17 \%$ to $42 \%), 19 \%(10 \%$ to $32 \%), 27 \%(12 \%$ to $46 \%), 7 \%(0 \%$ to $36 \%)$ and $20 \%(0 \%$ to $72 \%)$. Nearly half $(47 \%, 27$ inpatients on AS missed at least one day of AS dosing in Days 1-3. 


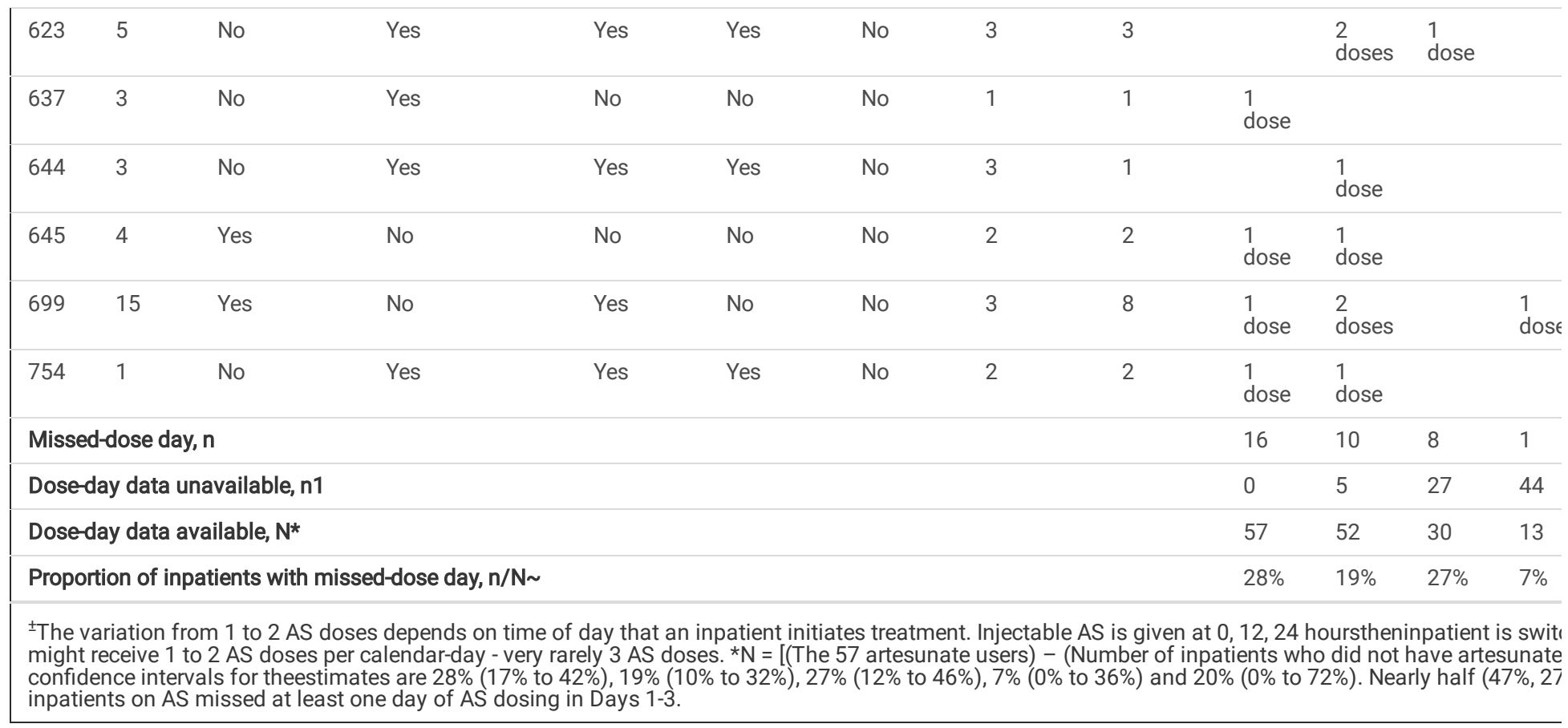

Quinine: One in five (18\%,5/28; $95 \% \mathrm{Cl}: 6 \%$ to $37 \%)$ of inpatients who initiated antimalarials with Q missed Day 1 doses based on calendar-day; $10 \%$ ( $2 / 20$; $95 \%$ Cl: $1 \%$ to $32 \%)$ missed Day 2 and $14 \%(1 / 7 ; 95 \%$ Cl: $0 \%$ to $58 \%)$ missed Day 3. One in five $(18 \%, 5 / 28 ; 95 \%$ Cl: $4 \%$ to $32 \%)$ inpatients missed at least one day of Q dosing in Days 1-3, see Table 5. 
Table 5

Missed dose-days of quinine injection among 28 hospitalized patients who received in-hospital intravenous quinine, Uganda, 2014

\begin{tabular}{|c|c|c|c|c|c|c|c|c|c|c|c|c|}
\hline fileno & $\begin{array}{l}\text { Length } \\
\text { of stay, } \\
\text { days }\end{array}$ & $\begin{array}{l}\text { Preadmission } \\
\text { antimalarial } \\
\text { use }\end{array}$ & $\begin{array}{l}\text { Admitting } \\
\text { Malaria } \\
\text { Diagnosis }\end{array}$ & $\begin{array}{l}\text { Discharge } \\
\text { Malaria } \\
\text { Diagnosis }\end{array}$ & $\begin{array}{l}\text { AL co- } \\
\text { prescribed }\end{array}$ & $\begin{array}{l}\text { Switched } \\
\text { to AL }\end{array}$ & $\begin{array}{l}\text { Doses } \\
\text { Prescribed }\end{array}$ & $\begin{array}{l}\text { Doses } \\
\text { Received }\end{array}$ & Day 1 & Day 2 & Day 3 & $\begin{array}{l}\text { Day } \\
4\end{array}$ \\
\hline 5 & 2 & No & Yes & Yes & 1 & & 3 & 2 & $\begin{array}{l}1 \\
\text { dose }\end{array}$ & $\begin{array}{l}1 \\
\text { dose }\end{array}$ & & \\
\hline 28 & 6 & Yes & Yes & Yes & 1 & & 3 & 3 & $\begin{array}{l}1 \\
\text { dose }\end{array}$ & & $\begin{array}{l}1 \\
\text { dose }\end{array}$ & $\begin{array}{l}1 \\
\text { dose }\end{array}$ \\
\hline 66 & 8 & Yes & No & No & 0 & & 3 & 1 & $\begin{array}{l}1 \\
\text { dose }\end{array}$ & & & \\
\hline 99 & 4 & Yes & Yes & Yes & 0 & & 3 & 3 & $\begin{array}{l}1 \\
\text { dose }\end{array}$ & $\begin{array}{l}1 \\
\text { dose }\end{array}$ & $\begin{array}{l}1 \\
\text { dose }\end{array}$ & \\
\hline 142 & 3 & No & Yes & Yes & 0 & & 3 & 3 & $\begin{array}{l}1 \\
\text { dose }\end{array}$ & $\begin{array}{l}2 \\
\text { doses }\end{array}$ & & \\
\hline 143 & 4 & No & Yes & Yes & 0 & & 3 & 1 & $\begin{array}{l}1 \\
\text { dose }\end{array}$ & & & \\
\hline 165 & 3 & No & Yes & Yes & 0 & & 3 & 2 & $\begin{array}{l}1 \\
\text { dose }\end{array}$ & $\begin{array}{l}1 \\
\text { dose }\end{array}$ & & \\
\hline 296 & 2 & Yes & Yes & Yes & 0 & & 3 & 2 & $\begin{array}{l}1 \\
\text { dose }\end{array}$ & $\begin{array}{l}1 \\
\text { dose }\end{array}$ & & \\
\hline 343 & 2 & Yes & Yes & Yes & 1 & & 3 & 1 & $\begin{array}{l}1 \\
\text { dose }\end{array}$ & & & \\
\hline 376 & 3 & No & Yes & Yes & 1 & & 3 & 2 & $\begin{array}{l}1 \\
\text { dose }\end{array}$ & $\begin{array}{l}1 \\
\text { dose }\end{array}$ & & \\
\hline 415 & 5 & Yes & Yes & Yes & 0 & & 3 & 3 & 2 doses & $\begin{array}{l}1 \\
\text { dose }\end{array}$ & & \\
\hline 416 & 3 & Yes & Yes & Yes & 0 & & 9 & 3 & $\begin{array}{l}1 \\
\text { dose }\end{array}$ & $\begin{array}{l}1 \\
\text { dose }\end{array}$ & $\begin{array}{l}1 \\
\text { dose }\end{array}$ & \\
\hline 417 & 4 & No & Yes & Yes & 0 & & 3 & 3 & $\begin{array}{l}1 \\
\text { dose }\end{array}$ & $\begin{array}{l}1 \\
\text { dose }\end{array}$ & $\begin{array}{l}1 \\
\text { dose }\end{array}$ & \\
\hline 419 & 3 & No & Yes & Yes & 1 & 0 & 3 & 3 & $\begin{array}{l}1 \\
\text { dose }\end{array}$ & $\begin{array}{l}2 \\
\text { doses }\end{array}$ & & \\
\hline 437 & 4 & No & Yes & Yes & 0 & & 6 & 2 & $\begin{array}{l}1 \\
\text { dose }\end{array}$ & $\begin{array}{l}1 \\
\text { dose }\end{array}$ & & \\
\hline 462 & 8 & No & Yes & Yes & 0 & & 3 & 3 & $\begin{array}{l}1 \\
\text { dose }\end{array}$ & $\begin{array}{l}2 \\
\text { doses }\end{array}$ & & \\
\hline 479 & 5 & Yes & Yes & Yes & 1 & 1 & 3 & 3 & $\begin{array}{l}1 \\
\text { dose }\end{array}$ & & $\begin{array}{l}1 \\
\text { dose }\end{array}$ & $\begin{array}{l}1 \\
\text { dose }\end{array}$ \\
\hline 484 & 4 & Yes & Yes & Yes & 1 & & 3 & 3 & $\begin{array}{l}3 \\
\text { doses }\end{array}$ & & & \\
\hline 489 & 3 & Yes & Yes & Yes & 0 & & 9 & 3 & $\begin{array}{l}2 \\
\text { doses }\end{array}$ & $\begin{array}{l}1 \\
\text { dose }\end{array}$ & & \\
\hline 491 & 2 & No & Yes & Yes & 0 & & 3 & 2 & $\begin{array}{l}1 \\
\text { dose }\end{array}$ & $\begin{array}{l}1 \\
\text { dose }\end{array}$ & & \\
\hline fileno & $\begin{array}{l}\text { Length } \\
\text { of stay, } \\
\text { days }\end{array}$ & $\begin{array}{l}\text { Preadmission } \\
\text { antimalarial } \\
\text { use }\end{array}$ & $\begin{array}{l}\text { Admitting } \\
\text { Malaria } \\
\text { Diagnosis }\end{array}$ & $\begin{array}{l}\text { Discharge } \\
\text { Malaria } \\
\text { Diagnosis }\end{array}$ & $\begin{array}{l}\text { AL co- } \\
\text { prescribed }\end{array}$ & $\begin{array}{l}\text { Switched } \\
\text { to AL }\end{array}$ & $\begin{array}{l}\text { Doses } \\
\text { Prescribed }\end{array}$ & $\begin{array}{l}\text { Doses } \\
\text { Received }\end{array}$ & Day 1 & Day 2 & Day 3 & $\begin{array}{l}\text { Day } \\
4\end{array}$ \\
\hline 598 & 9 & No & Yes & No & 0 & & 6 & 3 & & $\begin{array}{l}2 \\
\text { doses }\end{array}$ & $\begin{array}{l}1 \\
\text { dose }\end{array}$ & \\
\hline 633 & 3 & No & Yes & Yes & 1 & & 21 & 3 & $\begin{array}{l}2 \\
\text { doses }\end{array}$ & $\begin{array}{l}1 \\
\text { dose }\end{array}$ & & \\
\hline 674 & 2 & No & Yes & Yes & 1 & & & 1 & $\begin{array}{l}1 \\
\text { dose }\end{array}$ & & & \\
\hline 680 & 10 & No & Yes & Yes & 1 & 1 & 6 & 3 & $\begin{array}{l}2 \\
\text { doses }\end{array}$ & $\begin{array}{l}1 \\
\text { dose }\end{array}$ & & \\
\hline
\end{tabular}

${ }^{*} N=[($ The 28 quinine users) - (Number of inpatients without quinine dose-day data) $]$ or (28-n1); 95\% confidence intervals for the estimates are 18\% (6\% to $37 \%), 10 \%(1 \%$ to $32 \%), 14 \%$ (0\% to $58 \%)$. One in five $(18 \%, 5 / 28 ; 95 \%$ Cl: $4 \%$ to $32 \%)$ inpatients missed at least one day of Q dosing in Days $1-3$. 


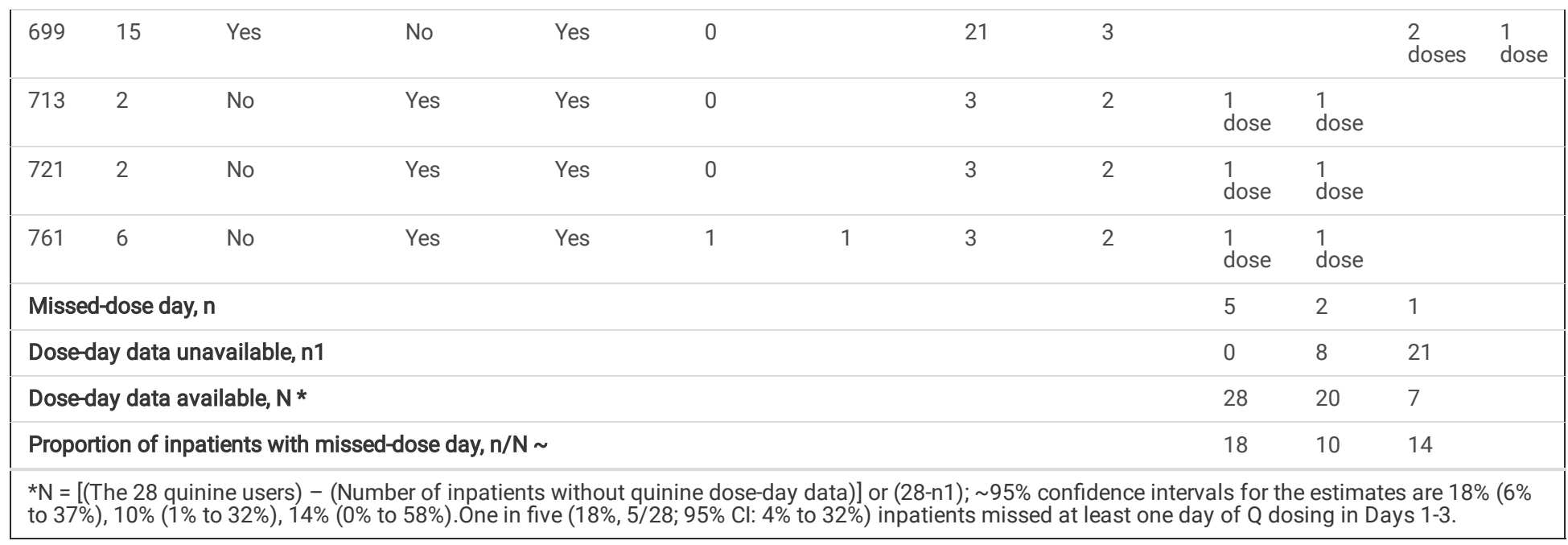

Artesunate vs. Quinine: The frequency of missing at least one day of antimalarials in Days 1-3 was significantly higher among inpatients who received AS vs. inpatients who received Q (difference of $29 \% ; 95 \% \mathrm{Cl}$ : $7 \%$ to $45 \% ; 1 \mathrm{df} ; \chi 2=6.7 ; P$-value $=0.010$ ).

Malaria-in-pregnancy: For both AS and Q, there was no significant difference in frequency of missed-dose days based on pregnancy-status, see Appendix.

\section{Mortality among inpatients who received in-hospital antimalarials}

Four of 100 inpatients who received in-hospital antimalarials died during hospitalization. All four inpatients had clinically-diagnosed malaria: microscopy was requested is three inpatients, but results were not available, see Box 2. Unconscious 88-year-old female of unknown HIV-status presented with a single admitting diagnosis of severe malaria and pulse rate of 98 beats per minute. She received a pre-referral intramuscular Q dose 23 hours preadmission and two Qdoses 11 hours apartafter admission. She died on Day 2 of hospitalization. The other three cases had multiple diagnoses, see Box 2.

\section{Patient-level risk-factors for missed Day 1 dosing of antimalarials}

Number of admitting diagnoses was a statistically significant risk-factor for missed Day 1 dosing of hospital-initiated antimalarialsbased on calendar-day (OR $=2.7,95 \%$ Cl: 1.53-4.54; P-value < 0.001), see Table 6. Similar results of missed Day 1 risk-factorwere obtained based on post-admission 24-hour-delay, see Table S1.Malaria-in-pregnancy was not significantly relatedto missed Day 1 dosing of antimalarials.

\section{Missed Day 1 dosing of hospital-initiated antimalarials versus length of hospital stay}

Nostatistically significant association was observedbetween missed Day 1 dosing of antimalarials and length of hospital stay $(\mathrm{OR}=1.1,95 \% \mathrm{Cl}$ : $0.91-1.27 ; P$ value $<0.396)$. Mean length of hospital stay for missed Day 1 cases was $4.7(S D=1.7)$ days versus $4.2(S D=2.5)$ days for non-cases. 
Table 6

Patient-level risk-factors for missed Day 1 dosing of administered antimalarials based on calendar-day delay among inpatients with an admitting malaria diagnosis, Uganda, 2014

\begin{tabular}{|c|c|c|c|c|c|c|c|c|c|}
\hline \multicolumn{10}{|c|}{ Missed Day 1 dosing of antimalarials by calendar-day, $n(\%) ;(N=83)$} \\
\hline \multirow[t]{2}{*}{ Factor } & \multicolumn{4}{|c|}{ Missed Calendar-Day 1 dosing } & \multicolumn{2}{|c|}{ Crude Analysis } & \multicolumn{3}{|c|}{ Adjusted Analysis } \\
\hline & Yes & No & Total, $[\% \text { col] }]^{\mathrm{a}}$ & $O R^{b}$ & $95 \%$ for $\mathrm{Cl}^{\mathrm{C}}$ & $P$-value & $\mathrm{OR}^{\mathrm{b}}$ & $95 \%$ for $\mathrm{Cl}^{\mathrm{C}}$ & $P$-value \\
\hline \multicolumn{10}{|c|}{ Antiretroviral therapy use } \\
\hline No & $15(20)$ & $59(80)$ & 74 [89] & 1.0 & & & 1.0 & & \\
\hline Yes & $5(56)$ & $4(44)$ & $9[11]$ & 4.9 & $1.17-20.6$ & 0.029 & 5.0 & $0.92-26.9$ & 0.062 \\
\hline \multicolumn{10}{|c|}{ Malaria microscopytest results available } \\
\hline No & $8(17)$ & $39(83)$ & $47[57]$ & 1.0 & & & 1.0 & & \\
\hline Yes & $12(33)$ & $24(67)$ & $36[43]$ & 2.4 & $0.87-6.82$ & 0.090 & 2.5 & $0.72-8.71$ & 0.146 \\
\hline \multicolumn{10}{|c|}{ Linear on number of working } \\
\hline Diagnoses & 20 & 63 & 83 & 2.6 & $1.56-4.35$ & $<0.001$ & 2.7 & $1.53-4.64$ & 0.001 \\
\hline
\end{tabular}

Box 1: Missed opportunity for hospital-initiated antimalarial treatmentfor four inpatients with malaria parasitaemia as confirmed by microscopy, Uganda.

Particulars Clinical notes

Patient 1 A 60-year-old female with unknown HIV-status, 6-year history of hypertension and type 2 diabetes mellitus (DM) presented with poorly controlled DM having defaulted on DM treatment for 8-months. Microscopy for malaria parasites was requested on the day of admission (Day 1). Results were returned on Day 1 with confirmed malaria parasitaemia. AL and paracetamol were prescribed on Day 2 but not dispensed. The patient was discharged on Day 3 without antimalarial treatment.

Patient 2 A 24-year-old female with unknown HIV-status was referred from a clinic where she had been treated for suspected malaria and typhoid with no improvement. She presented with poorly treated malaria and a request for microscopy for malaria parasites was made on Day 1 . Results were returned on Day 2 with confirmed malaria parasitaemia. AL and paracetamol were prescribed on Day 2 but not dispensedand the patient was discharged on Day 2 without antimalarial treatment.

Patient 3 A 44-year-old HIV-negative male was transferred from a referral hospital. He presented with an admitting diagnosis of chronic lymphocytic leukaemia and confirmed malaria parasitaemia by microscopy. No fresh request for malaria microscopy was made during the current admission. The patient did not receive any antimalarial treatment prescription and/or administration both prior to admission and throughout the current hospitalization. He was transferred to Uganda Cancer Institute on Day 3.

Patient 4 A 43-year-old HIV-positive female with history of DM and receiving second-line antiretroviral therapy, ART (tenofovir, lamivudine, lopinavir/ritonavir) and co-trimoxazole presented with an admitting diagnosis of colon cancer. Microscopy for malaria parasites was requested on Day 2 and results were returned the same day with confirmed malaria parasitaemia. No antimalarial treatment was prescribed, dispensed or administered during hospitalization though the patient continued to receive her ARTP and co-trimoxazole. The patient was transferred to Uganda Cancer Institute on Day 17. 
Box 2: Mortality of four inpatients who received in-hospital antimalarial treatment, Uganda.

\section{Particulars Clinical notes \\ Quinine One inpatient who received Q during admission died in hospital.}

Patient 1-Q An 88-year-old female of unknown HIV-status presented with a single admitting diagnosis of severe malaria which manifested with fever, chills and unconsciousness. She was referred from a clinic for further management after receiving an initial intramuscular dose of quinine ( 23 hours prior to the current admission). Her vitals on admission were: pulse rate ( 98 beats per minute); blood pressure (116/63 $\mathrm{mmHg}$ ); temperature $\left(35.9^{\circ} \mathrm{C}\right)$. Microscopy for malaria parasites was requested on admission (Day 1$)$ but the results were not returned by Day 2. She received 2-doses of $Q$ which were administered 11 hours apart, the first dose being 2 hours after admission on Day 1 . The patient died on Day 2 of hospitalization.

\section{Artesunate Two inpatients who received AS during admission died in hospital. None of the two inpatients presented with either an admitting or a} discharge malaria diagnosis:

Patient 1-AS A 20-year-old female of unknown HIV-status was admitted with suspected severe sepsis of chest focus, bacterial pneumonia, urinary tract infection (UTI), salmonellosis and acute gastroenteritis. Microscopy for malaria parasites was requested on Day 1 but the results were not returned. She missed Day 1 dosing of AS and subsequently received 4 doses of AS. Her discharge diagnoses were UTI, pneumonia and salmonellosis. She died on Day 4.

Patient 2-AS A 24-year-old HIV-positive female presented with severe immunosuppression, sepsis, disseminated tuberculosis and/or tuberculous meningitis, atypical measles syndrome and toxoplasmosis. Microscopy for malaria parasites was not requested on admission. She received 2 doses of AS and never missed Day 1 dosing. Her discharge diagnosis was severe immunosuppression. She died on Day 10.

Artemether + $\quad$ One inpatient who received AL in hospital died.

Lumefantrine

Patient 1-AL A 23-year-old HIV-positive female presented with working diagnoses of immunosuppression, malaria, septicaemia, urinary tract infection and anaemia. Microscopy for malaria parasites was requested on admission (Day 1) but the results were not returned. Duocotexcin (DP) was prescribed on Day 1 but was neither dispensed nor administered. One dose of AL was administered on Day 3. Her discharge diagnoses were immunosuppression and malaria. She died on Day 6.

\section{Discussion}

Malaria microscopy was requested in $77 \%$ of inpatients with an admitting malaria diagnosis, similar to estimates for the public sector (80\%) in moderate- to high-transmission countries in sub-Saharan Africa (SSA) [1]. Unfortunately, only half the microscopy results were available to guide appropriate antimalarial treatment. Thus, despite decent microscopy rates, healthcare professionals still rely on clinical judgement to treat half the suspected malaria cases. Clinical judgement increases the risk of unnecessary antimalarial treatment and, in turn, depletes antimalarial stocks for inpatients who truly need them; and increases the incidence of associated adverse drug reactions and drug resistance [2]. Seven in ten inpatients with suspected non-pregnancy-related malaria tested negative for malaria and would therefore not need antimalarials; compared with only two in ten inpatients with suspected malaria-in-pregnancy. The value of a confirmed malaria diagnosis depends on prompt availability of parasitology results and whether the clinician uses the results to decide how to manage the patient. Malaria negative test-results as confirmed by microscopy - the gold standard - should prompt clinicians to examine patients for other causes of illness and treat them accordingly [2]. However, the interpretation of negative microscopy results should take into account the high rates of antimalarial pre-treatment, which was as high as one in three admitted patients with suspected malaria in this patient cohort. A rapid diagnostic test (RDT), in addition to microscopy, could be used to detect the HRP2 malaria antigen in patients who recently received antimalarials and whose blood films are, thus, likely to show no malaria parasitaemia [2]. RDTs can give positive results for up to 1-month after parasite clearance [2].

One in six cases of confirmed malaria did not receive antimalarials during the current hospitalization, which raises concern over the safety of inpatient care at this tertiary care hospital. Poor coordination between the laboratory and clinicians is likely, which is exacerbated by high inpatient loads of up to 80 admissions in wards with official bed capacity of 54 [3]. Introducing an integrated electronic health record (EHR) system to track inpatient care could significantly improve the flow of information between different hospital departments and, in so doing, promote efficient clinical management of inpatients [12].

One in four inpatients who received at least one in-hospital dose of prescribed antimalarials missed the first day of their antimalarials, which is relatively frequent. Also, monotherapy and incomplete dosing primarily associated with injectable AS and Q were common, possibly fuelled by observed disparities in prescribed, dispensed and administered antimalarials - similar to observations made elsewhere [8, 10]. Possible reasons for these system lapses include; i) drug stock-outs, ii) poor communication between clinician and patient/caregiver and, iii) work overload [3]. The hospital should improve its stock forecasting for in-demand antimalarials, continue to promote intern-pharmacist-led bedside dispensing to reduce the clinicians' workload during drug administration and improve supervision of junior and mid-level clinicians to promote accountability to inpatients and the hospital [3].

Each additional admitting diagnosis increases by more than two-fold the odds of missed Day 1 dosing of prescribed antimalarials, which underlines the need for prompt availability of malaria test-results to promote the timely initiation of antimalarials. Prompt and complete antimalarial treatment rapidly eliminates malaria parasites from a patient's bloodstream [13]. Patients with severe malaria should access timely appropriate antimalarials, avoid antimalarial monotherapy and complete full courses of prescribed antimalarials to promote therapeutic success, reduce malaria-related morbidity and mortality, and prevent the emergence and spread of drug resistance [7-9]. 
Inpatients with an admitting malaria-in-pregnancy diagnosis seemed more likely to have a microscopically-confirmed malaria diagnosis than inpatients with other admitting malaria diagnoses. This comparative advantage at diagnosis did not translate into better antimalarial treatment because no pregnancyrelated difference was observed in the prescription, dispensing and administration of antimalarials. Improvement in the antimalarial medication-use-cycle should target systemic weaknesses.

Unlike Q, the hospital frequently encounters drug stock-outs of in-demand, free-of-charge AS and AL, which inpatients must purchase from private community pharmacies to prevent lapses in prescribed treatment. Drugs bought from private community pharmacies are not recorded as dispensed in the hospital register [3], which explains why the reported number of inpatients with administered AS and AL exceeds the number of inpatients to whom these two drugs are dispensed. AS and AL are more in demand than Q because; i) AS is the drug of choice for its faster parasite clearance, less tedious administration regime, and safer profile and, ii) AL is administered after both injectable AS and Q as the continuation of antimalarial treatment in severe malaria [2].

Death could be attributed to severe malaria and/or quinine-related treatment in the 88-year-old female with a single admitting severe malaria diagnosis. The caveat to this malaria-related attribution is malaria based on clinical judgement only (in the absence of microscopy results), unknown HIV-serostatus, advanced age, unknown random blood sugar levels and other comorbidities - especially cardiovascular comorbidities. That notwithstanding, Q was poorly administered at intervals of 25 hours (between first and second doses) and 11 hours (between second and third doses). Yet, 8-hourly intervals of injectable Q administration for at least 24 hours are recommended until the patient is able to take oral medication [2]. The unconsciousness manifested in this inpatient is a known key sign of hypoglycaemia in severe falciparum malaria and carries a high risk of mortality [2]. Unfortunately, hypoglycaemia can result from both severe malaria and quinine-induced hyperinsulinaemia. Thus, blood sugar levels should be checked frequently in severe malaria inpatients who receive Q [2]. Also, this inpatient had tachycardia which could have resulted from Q use and/or hypoglyaemia. With hindsight, this elderly inpatient should have been treated with injectable AS instead, although the frequent unavailability of in-demand AS, and its associated higher cost, often dictates treatment with Q. This fatal case of suspected severe malaria underpins the need for the rapid turnaround of microscopy test-results and the hospital's investment in routine random blood sugar testing to improve the clinical management of inpatients with severe malaria.

In conclusion, half the malaria microscopy results were not available to guide the clinical management of malaria despite that the rate of testing was high. Laboratory services should improve to promote the treatment of malaria based on confirmed diagnosis as opposed to clinical judgement only, which could reduce the unnecessary use of antimalarials. System-level improvement is required in the procurement, prescription, dispensing and administration of antimalarials to curb the rampant missed opportunities for antimalarial treatment, monotherapy especially with injectable antimalarials and delayed/missed antimalarial doses.

The study's limitations have been reported elsewhere [3]. Briefly, the study was conducted at Uganda's National Referral and Teaching Hospital and the results might not be generalizable to facilities with lower calibres of inpatient care. Also, antimalarials that were purchased from private community pharmacies were not documented as dispensed in the hospital register so we obtained this dispensing information by interviewing the inpatients and/or their caregivers [3].

\section{Declarations}

\section{Ethics approval and consent to participate:}

Ethical approval was granted by the School of Medicine Research and Ethics Committee, Makerere University College of Health Sciences (REC REF No. 2011 113), Mulago Hospital Research and Ethics Committee (MREC 253), and Uganda National Council for Science and Technology (HS 1151). All participants gave written informed consent.

\section{Consent for publication:}

Consent to publish this work was sought during the informed consent process.

\section{Availability of data and materials:}

The dataset for this publication is available on reasonable request from the corresponding author.

\section{Competing interests:}

SMB. holds GSK shares. RK and CK have nothing to declare.

\section{Funding:}

The study received grant support from Wellcome Trust through the Training Health Researchers into Vocational Excellence in East Africa programme (grant number 087540); and the African Population and Health Research Centre (2013-2015 ADF 006).

\section{Authors' contributions:}


RK conceived the study, supervised data collection and conducted data analysis. RK, SMB and CK designed the study, participated in interpretation of results and manuscript writing. All authors read and approved the final draft of the manuscript.

\section{Acknowledgements:}

The authors are grateful to the study participants who consented; and the administration of Mulago Hospital which permitted the study.

\section{References}

1. World Health Organization. World Malaria Report 2019. In: Organization WH, editor. Geneva: World Health Organization; 2019.

2. World Health Organization. Guidelines for the Treatment of Malaria. Treatment of Severe Malaria 2015 [cited 202030 April]; 3rd Edition:[Available from: https://www.ncbi.nlm.nih.gov/books/NBK294445/.

3. Kiguba R, Karamagi C, Bird SM. Extensive antibiotic prescription rate among hospitalized patients in Uganda: but with frequent missed-dose days. The Journal of Antimicrobial Chemotherapy. 2016;71(6):1697-706.

4. Dondorp A, Nosten F, Stepniewska K, Day N, White N. Artesunate versus quinine for treatment of severe falciparum malaria: a randomised trial. Lancet. 2005;366(9487):717-25.

5. Dondorp AM, Fanello Cl, Hendriksen IC, Gomes E, Seni A, Chhaganlal KD, et al. Artesunate versus quinine in the treatment of severe falciparum malaria in African children (AQUAMAT): an open-label, randomised trial. Lancet. 2010;376(9753):1647-57.

6. Sinclair D, Donegan S, Isba R, Lalloo DG. Artesunate versus quinine for treating severe malaria. The Cochrane Database of Systematic Reviews. 2012; (6): CD005967.

7. Amboko BI, Ayieko P, Ogero M, Julius T, Irimu G, English M. Malaria investigation and treatment of children admitted to county hospitals in western Kenya. Malaria Journal. 2016;15(1):506.

8. Ampadu HH, Asante KP, Bosomprah S, Akakpo S, Hugo P, Gardarsdottir H, et al. Prescribing patterns and compliance with World Health Organization recommendations for the management of severe malaria: a modified cohort event monitoring study in public health facilities in Ghana and Uganda. Malaria Journal. 2019;18(1):36.

9. Riley C, Dellicour S, Ouma P, Kioko U, ter Kuile FO, Omar A, et al. Knowledge and Adherence to the National Guidelines for Malaria Case Management in Pregnancy among Healthcare Providers and Drug Outlet Dispensers in Rural, Western Kenya. PloS One. 2016;11(1):e0145616.

10. Dlamini SV, Kosgei RJ, Mkhonta N, Zulu Z, Makadzange K, Zhou S, et al. Case management of malaria in Swaziland, 2011-2015: on track for elimination? Public Health Action. 2018;8(Suppl 1):3-7.

11. StataCorp. Stata Statistical Software: Release 12. College Station. TX: StataCorp LP; 2011.

12. Schiza EC, Panos G, David C, Petkov N, Schizas CN. Integrated Electronic Health Record Database Management System: A Proposal. Studies in Health Technology and Informatics. 2015; 213: 187-90.

13. World Health Organization. Compendium of WHO malaria guidance - prevention, diagnosis, treatment, surveillance and elimination. In: Organization WH, editor. Geneva: World Health Organization; 2019.

\section{Supplementary Files}

This is a list of supplementary files associated with this preprint. Click to download.

- Appendix14May2020.docx 